\title{
IMSEC EVENTS BASH -AN ANDROID APPLICATION
}

\author{
Ekta Singh Kushwaha \\ Department of Computer Science \&Engineering \\ IMS Engineering College, Ghaziabad \\ Uttar Pradesh, India
}

\begin{abstract}
In the modern era of digitalization smartphones are playing an important role in everyone's life. Smartphone's software is taking care of the needs of every individual in the form of virtual assistants. In the context of students, they provide incredible facilities such as online classes, online exams, tracking syllabus, etc. In this era of competition, it is necessary to keep an eye on important notices of different activities that are circulated by colleges or corresponding educational institutes because missing any notification may lead to loss of a great opportunity. Sometimes manual notice board fails in propagating the news or notices properly. This paper intends to solve the problems of propagating news and information and also alleviate the problem of traditional event managing procedures such as lots of paperwork, long queues at the registration desk, or offline payment issues. The objective of this project is to develop an android application which provides important news about events through the notification alerts. Moreover, students will be able to manage their event participation, such as reserving their seats in events, registering at the event site, and so on. More importantly, this application uses email verification to provide an easy way to verify the participant's identities for the authentication process.
\end{abstract}

Keywords-Kotlin, XML, Firebase, Android Studio, Validation, Notification, Events.

\section{INTRODUCTION}

Nowadays, mobile phones have become popular with everybody since it is very convenient to use. Everyone uses mobile phones daily to connect with friends, family, and others. It has become a huge part of our life. It provides a wide range of information in every field such as education, courses, news, movies, etc. Smartphones are the most common computational device that is possessed by most of the students nowadays, which motivates them to create an android application that can spread information about events easily anytime, anywhere to every student.

This paper is about the "IMSEC EVENT BASH" android application which is one of the creations of motivation.

\author{
Vaishali Gupta \\ Department of Computer Science \&Engineering \\ IMS Engineering College, Ghaziabad \\ Uttar Pradesh, India
}

"IMSEC EVENTS BASH" android application helps the users to participate in events through online notices of the institution. It is a type of online notice board where students can easily get instant notifications. IMSEC EVENTS BASH brings the notices to the events dashboard where students can not only read notices but also immediately react and respond to them using a contact option provided in the application for any query. The user can also enroll in the events through its user interface. The user will receive notifications regarding college activities like placements, seminars, conferences, sports, and cultural events in their notification section. This innovative app allows the students to get the notifications about the events directly after downloading the application in their android phones and then logging themselves into the application for registration of the events \& paying the required amount after registration. Users can pay the required amount by using any UPI Id. Some of the supported payment apps are google pay, amazon pay, Paytm, BHIM, Phonepe. After a successful registration confirmation email is sent to the user's registered email Id. They can also give suggestions and can also ask queries while registering for the event through the portal provided in the application itself. Admin can delete the past events, Add new events, provide the details about any particular event and whom to contact for further more details of that corresponding event.

Another important feature is that special permissions are provided based on the designation. Only Principal, HOD, and faculties are allowed to add new event details. This paper aims to provide the app features that help the students to overcome the problems of not getting the notices in time.

\section{LITERATURE SURVEY}

This paper proposes the idea of implementing an android application that uses technologies like Android studio, Kotlin, XML, along with the firebase database. A lot of previous work related to this topic has been carried out. This paperwork suggests the idea to improve the previous ideas by reducing the charge of database maintenance and time consumption in app development by using Kotlin. Durelli et al (2018) explained Kotlin as a multi-paradigm language, supporting both object-oriented and functional programming paradigms, 
i.e., it allows developers to combine them, as with most modern languages nowadays [1]. According to Oliveira et al (2020) Google has announced Kotlin as one of the officially supported languages for Android app development [2]. Google itself has mentioned some effective reasons for choosing Kotlin as an android app development language such as "it is concise, expressive, and designed to be null-safe".XML stands for Extensible Markup Language it is specially designed for the Internet. According to Jiang et al (2019) the focus of XML is on managing the data itself, not on the style of the data, and the display of the data is left to another technique [8]. In the reference of works on android app development, based on the interaction between college and students, there had been a lot of significant works like online attendance system, Online assignments submission, Online Assessments, etc. This paper can be termed as a new bead in the digitalization chain of the academic curriculum. As, Jacksi et al (2018) explained their work in the form of a website that has been designed to ensure up to date information of the college events, to improve the college event management record, and to enlarge the interest of students towards college events [3]. Also, Honglei et al (2016) and Akhila et al (2013) designed a student tracker system that has been developed for the android platform. It monitors the online attendance of students [4,5]. Kokate et al (2014) developed an ERP system to maintain the records of students in colleges and universities [6]. Singh et al (2016) described their work in the form of a web portal for the Student Management system that has been developed using HTML and SQL [7]. Other platforms like google classrooms, class chat rooms, etc are also popular for college-student virtual interaction. The current paper is devoted to support the previous works to make the information easily reachable.

\section{TECHNOLOGY STACK}

\section{A. Android Studio-}

Android studio is a framework to develop android applications based on IntelliJ IDEA was released in 2014 which replaced Eclipse Android development tools. Java was replaced by Kotlin in May 2019 which is preferred by google's language for application development. Android studio can be downloaded on Mac, Linux, Windows which provides robust static analysis and quickly fixes issues like security, performance, and correctness. Its Instant app run is less time consuming to rebuild the apk, installations made, and assemble changes immediately in apps. This framework has a great feature called Fast Emulator which is entirely like android phones for testing applications. It has a Visual Layout Editor which builds layout designs by adding distinct attributes, can be connected with Firebase database, and supports Kotlin. Android studio can build applications for various screen sizes as well as wear and gear devices.

\section{A. AndroidOperatingSystem-}

The android operating system is a free and open-source software which is based on Linux Kernel, initially developed by Android Inc. Later in 2005, it was acquired by Google. It was originally designed for smartphones and tablet computers but today an android os is also used in smart televisions, wristwatches, etc. Android applications can be implemented in Java, Kotlin programming language using the Android Software Development Kit (SDK). The Android SDK provides the tools and libraries necessary to begin developing applications that run on Android-powered devices. Different languages provide their platform to develop android applications like python provides kiwi, Java is used as an official language, $\mathrm{C}++$ can be used with Native Development Kit, Javascript can be used with PhoneGap framework and so on.

\section{B. Extensible Markup Language-}

XML is used to design UI layouts and screen elements. The $\mathrm{XML}$ file is saved with the .Xml extension. $\mathrm{Xml}$ is designed UI layouts just as the same way to create web pages in Html. Basically in the XML, two application programming interfaces are DOM and SAX.

\section{DOM}

The Document Object Model is a programming API for HTML and XML documents. These documents have a hierarchy of informational units called nodes; DOM is a standard way of describing those nodes and the relationship between them. DOM aims to provide a standard programming interface that can be used in different environments and applications. It can be implemented with any language. It defines the logical structure of documents, which is similar to a tree and the way of accessing \& manipulating documents. The methods of DOM allow programmatic access to the tree as one can change the structure, style, or content of a document. Nodes of XML documents have their attached event handlers so that once an event is triggered, the event handlers get executed. The DOM supports navigation in any direction and allows for modifications

\section{SAX}

SAX stands for Simple API for XML. It is an event-driven online algorithm for parsing XML documents. It provides a mechanism for fast reading of data from an XML document that is an alternative that is provided by DOM. The working of SAX parser is different from the DOM parser. A SAX parser only needs to report each parsing event as it happens, and discard almost all the information once reported. It uses a callback function to inform the clients of the XML document structure.SAX parser can parse files of any size as it neither load any XML document into memory nor creates any object 
representation of the XML document so the memory usage is generally much smaller and does not increase.

\section{Kotlin-}

Kotlin is a free, open-source, general-purpose programming language that is designed for Java Virtual Machine and developed by JetBrains. It is an Object-oriented programming language and is a better language than Java but still, both can exchange the information and use it. Kotlin is concise and reduces the amount of code, is safe due to its avoidance of errors such as null pointer exceptions, is interoperable and tool friendly.

\section{Firebase}

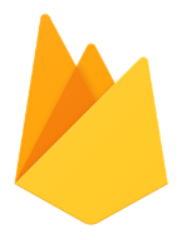

Firebase as shown in Fig. above assists web and mobile platforms to build high-quality applications. It was developed in 2011 by Firebase, Inc. which was then obtained by Google in 2014. Firebase provides Backend as a service and is widely used by developers for the reason that it provides varied functionalities one of being popular is a Real-time database that synchronizes application data over android, IOS, web devices, and stores it on its another feature Firebase's cloud.

The real-time database is a cloud-hosted database that gives secure access to the database to its users regardless of network quality and saves developers to invest high in hardware storage and the physical space occupied by data. Firebase is backed by Google cloud storage which offers advanced security, cost-effective, and sharing potentiality. Firebase Authentication is the feature that gives security to Apps which confirms clients over its application. It supports various signin providers such as Password, Email, phone number, Google, Twitter, Facebook, etc. Firebase cloud messaging also known as Google cloud messaging that provides messaging and notification at zero expense for Android, IOS, and web applications make Firebase superior to other databases.

Firebase services offer features that help to build better apps, improve app quality, have incredible built-in analytics, hosting, and even offer you to earn money and make growth in business.

\section{Methodology}

The methodology is accomplished in two modules: First is the User Module and the Second is the Admin Module.

\section{A. User Module-}

- Enter Login Credentials- In this User opens the installed application and enters his/her login credentials.

- Event Details- When the user successfully login, a list of events is displayed containing all information about it.

- Registration- Users can register into an event according to his/her interests by tapping onto the Register option and thereby giving details.

- Email Notification- On Successful registration, the User gets the notification about the registration in their registered email id.

- Payment- Payment is done as per the requirement of an event by using GooglePay, AmazonPay, Paytm, PhonePe, UPI.

- Event's History- In this Users can see who has participated in the event and can also share with other people.

- Event Notification- The user gets the notification about the new events.

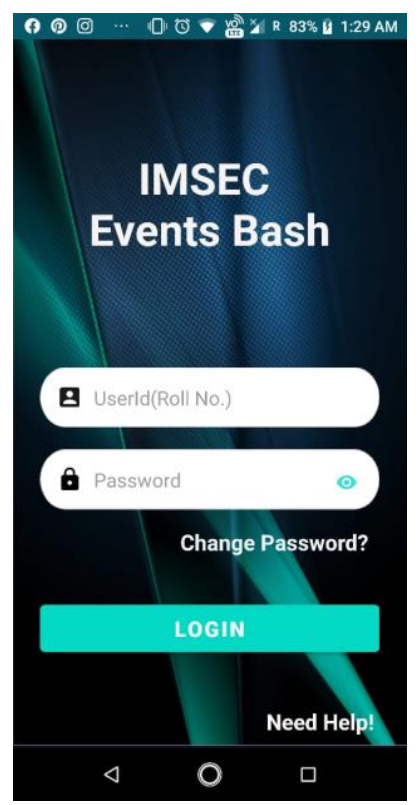

(a)

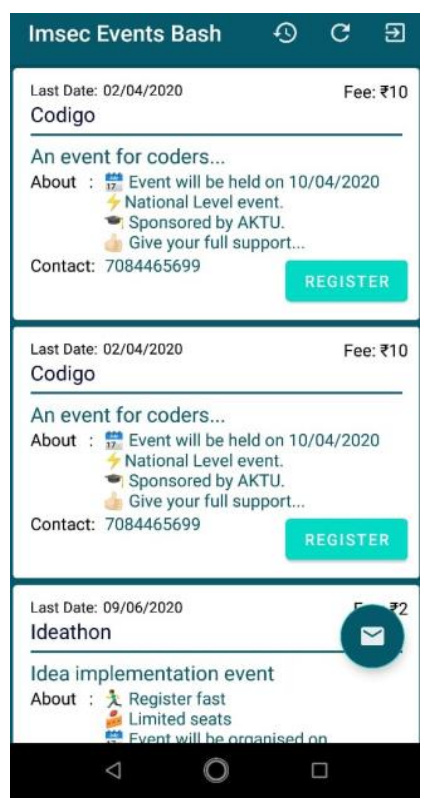

(b)
Fig. 1. (a) Login page (b) List of events 
International Journal of Engineering Applied Sciences and Technology, 2020

Vol. 5, Issue 2, ISSN No. 2455-2143, Pages 282-287

Published Online June 2020 in IJEAST (http://www.ijeast.com)

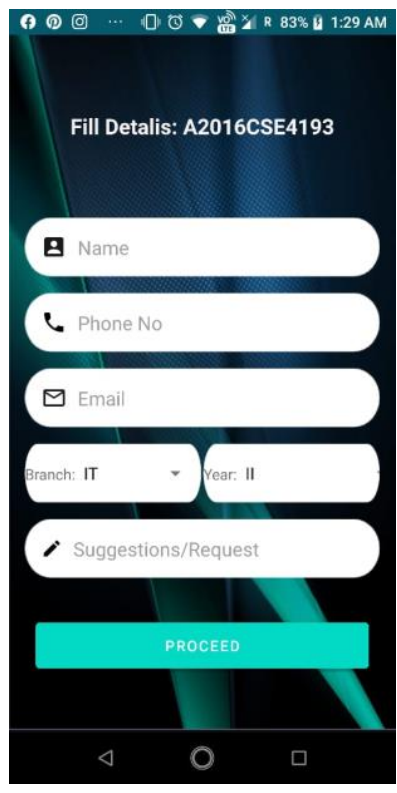

(a)

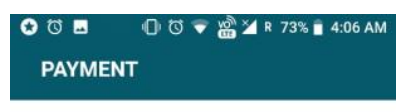

IMSEC Events Bash

\section{Amount to pay: ₹10}

Order id:A2016CSE4193Codigo

\section{SELECT APP BELOW}

Your Payment Is Safe And Secure

Any Query?

Contact At: Iimseceventsbash@gmail.com'
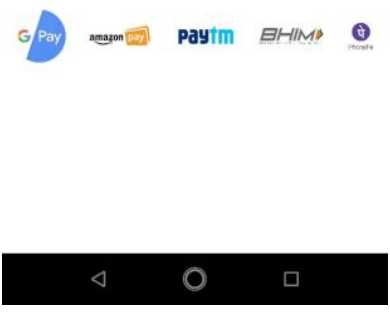

(b)

Fig. 2. (a) Registration Details (b) Payment page

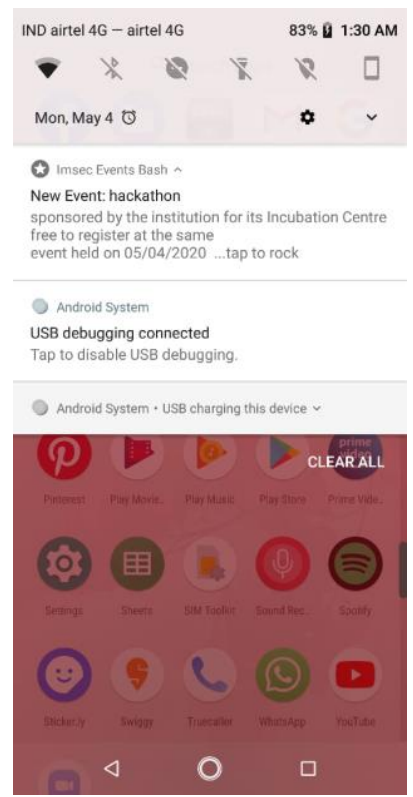

(a)

Fig. 3.(a) Event notification on a mobile phone.

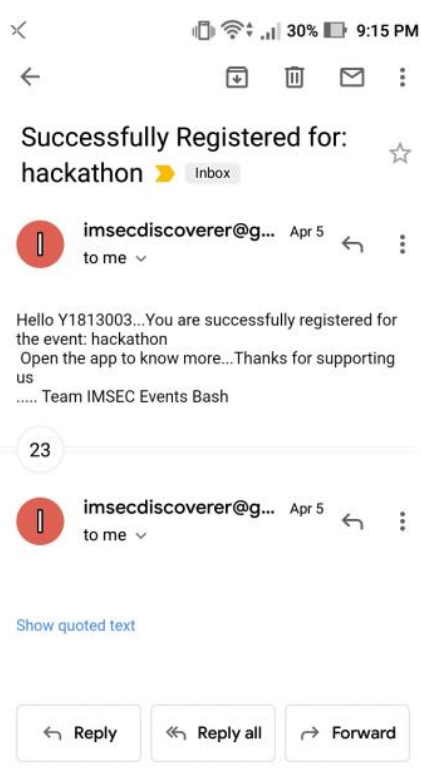

(b)

Fig 4(g) describes the whole procedure at the student module end as when the student login with their authenticated credentials, a list of events will open and then the student can register in any particular event according to their field of interest and can confirm their seat after the payment through email. Student can also share their event details with anyone.

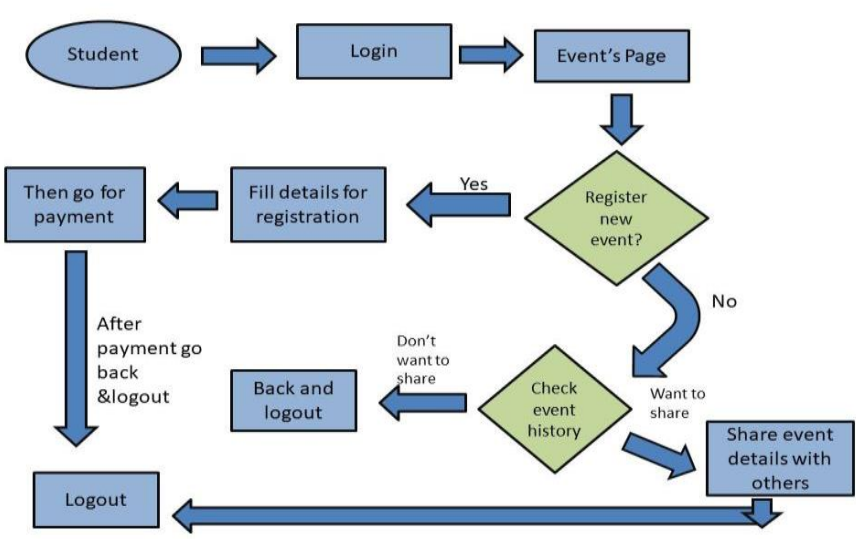

(g)

Fig.4(g) Flowchart of the student module 


\section{B. Admin Module-}

As shown in the Fig.6.(a) depicts the flowchart of the admin module. Admin login in the app with the particular Id and password. On successful login, admin can add new events by filling the details of events, delete or disable past events, share the details who have registered, can contact the developer, check the history of the events, and check the status of every user. Admin can log out of the app at any time.

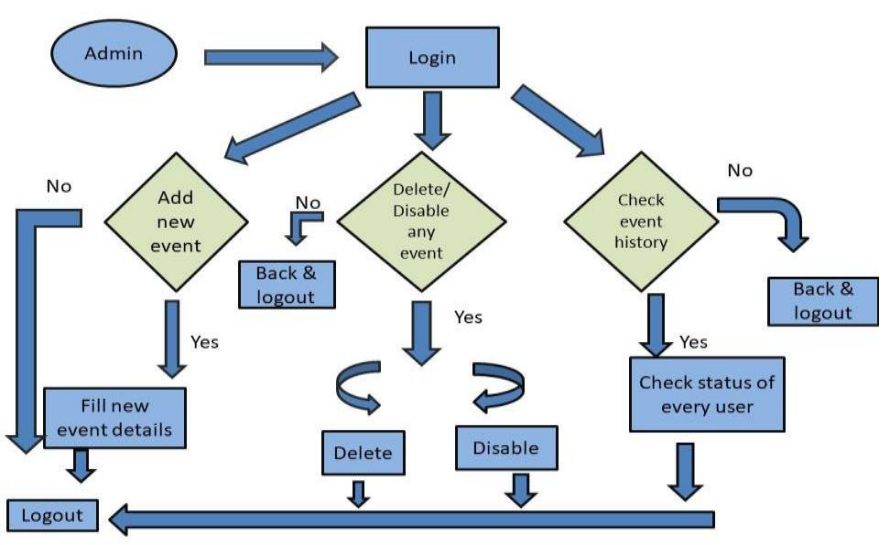

(a)

Fig.6(a) Admin Module Flowchart

This module can perform the following functionalities :

- Add New Event - Admin can add new events.

- Delete Event- Admin can delete events.

- Share details- Admin can also share details of events with other people.

- Events history- Admin can also see the history of events just like users.

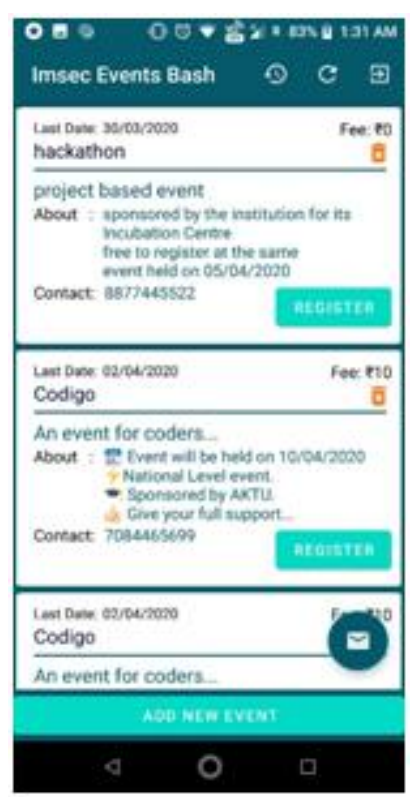

(b)

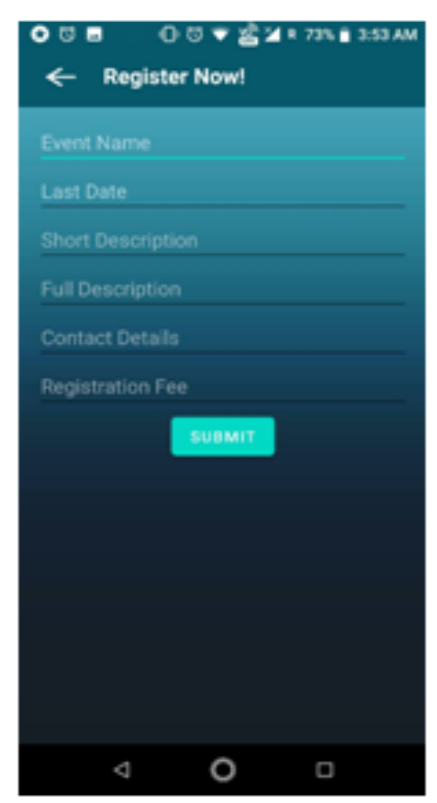

(c)

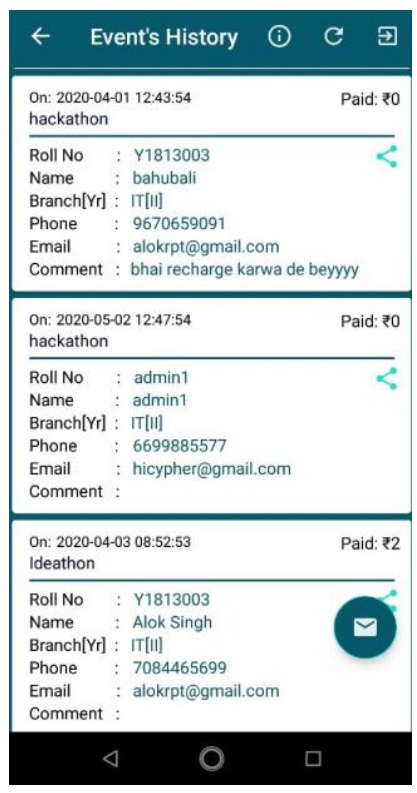

(d)

Fig. 6 (b)Events history (c) Add and delete new event (d) Event's History

\section{CONCLUSION}

This project has tried to analyze the existing systems which are currently used by colleges to notify students about the activities which are manual, time-consuming, and require too much manpower. To overcome these issues, a system has been proposed which is an android application that aims to provide notification of events to students anytime, anywhere easily.

This paper is an effort to implement the following features and to eliminate corresponding issues:

- To reduce the difficulties in the participation procedure of the events.

- To bind the students with cultural as well as sports activities so that they can learn other activities too.

- To promote digital transactions using UPI.

- To spread the details of the events among students.

- To send alert notifications to the users to increase the number of participants in the events.

- To reduce the overhead to check manual notice boards.

\section{ACKNOWLEDGMENT}

We are grateful to all those with whom I have had the pleasure to work during this project. We are especially indebted to our Guide Ms. Juhi Chaudhary Ma'am who has been supportive during my project and who worked actively to pursue this project goal. We also take this opportunity to express deep gratitude to our friends for their support and inspiration that help me accomplish this work through its various stages. We are responsible for providing valuable information provided to faculty 


\section{International Journal of Engineering Applied Sciences and Technology, 2020 \\ Vol. 5, Issue 2, ISSN No. 2455-2143, Pages 282-287 \\ Published Online June 2020 in IJEAST (http://www.ijeast.com)}

members of the Computer Science And Engineering Department at IMSEC Ghaziabad in their respective fields. We are grateful for their cooperation during our appointment. Finally, thanks to the continued encouragement of our faculty members, this work would not have been possible without you.

\section{REFERENCES}

1. M. Flauzino, J. Verssimo, R. Terra, E. Cirilo, V. H. S. Durelli, and R. S. Durelli. "Are you still smelling it?: A comparative study between Java and Kotlin language". 2018. In Proceedings of the VII Brazilian Symposium on Software Components, Architectures, and Reuse (SBCARS '18). ACM, New York, NY, USA, 23-32. DOI: https://doi.org/10.1145/3267183.3267186.

2. Oliveira, V., Teixeira, L., \& Ebert, F. (2020). On the Adoption of Kotlin on Android Development: A Triangulation Study. 2020 IEEE 27th International Conference on Software Analysis, Evolution, and Reengineering doi:10.1109/saner48275.2020.9054859

(SANER).

3. Jacksi, Karwan Ibrahim, Falah \& Zebari, Shahab. (2018). Student Attendance Management System. International Journal of Engineering and Technology. 6. 49-53. 10.21276/sjet.2018.6.2.1.

4. Honglei, Ren \& Song, You \& Yang, Siyu. (2016). An automated student attendance tracking system based on voiceprint and location. 10.1109/ICCSE.2016.7581583.

5. Akhila, K., Prathyusha, B., Pavankumar, M., \& Amrutha, M. (2013). A Novel Approach Of Mobile Based Student Attendance Tracking System Using Android Application. International journal of engineering research and technology, 2.

6. Kokate, Shrikant. (2014). E-College: An ERP for Educational Institute.

7. Singh, Thakur \& Rao, Dr. J Durga. (2016). A STUDY OF WEB PORTAL FEATURES AS A KNOWLEDGE MANAGEMENT SYSTEM IN SCHOOL EDUCATION. Review of Research ISSN No : 2249-894X. 5. 1-4.

8. Jiang, Li. (2019). Application Research of XML Parsing Technology Based on Android. Journal of Physics: Conference Series. 1314. 012213. 10.1088/17426596/1314/1/012213.

9. Kadam, Anilkumar \& Singh, Aradhana \& Jagtap, Komal \& Tankala, Srujana. (2017). Mobile Web Based Android Application for College Management System. International Journal Of Engineering And Computer Science. 10.18535/ijecs/v6i2.07.

10. Ubaya, Huda. (2012). Design of Prototype Payment Application System With Near Field Communication (NFC) Technology based on Android. Computer Engineering and Applications Journal (ComEngApp). 1. 1-12. 10.18495/comengapp.v1i1.1.

11. Khawas, Chunnu \& Shah, Pritam. (2018). Application of Firebase in Android App Development-A Study. International Journal of Computer Applications. 179. 4953. 10.5120/ijca2018917200.
12. Moroney, Laurence. (2017). The Firebase Realtime Database. 10.1007/978-1-4842-2943-9_3.

13. Moroney, Laurence. (2017). Firebase Cloud Messaging. 10.1007/978-1-4842-2943-9_9.

14. Bose, Subham. (2018). A COMPARATIVE STUDY: JAVA VS KOTLIN PROGRAMMING IN ANDROID APPLICATION DEVELOPMENT. International Journal of Advanced Research in Computer Science. 9. 41-45. 10.26483/ijarcs.v9i3.5978.

15. Kanagasabai, Thiruthanigesan \& Thiruchchelvan, Nagarathnam. (2017). Data Verification and Validation Process in the Management System Development. Middle East Journal of Scientific Research. 25. 902-911. 10.5829/idosi.mejsr.2017.902.911.

16. Esmaeel, Hana \& Esmaeel, Hana. (2015). Apply Android Studio (SDK) Tools. Baghdad Science Journal. Vol.7.

17. Yener, Murat \& Dundar, Onur. (2017). Android Application Development With Android Studio. 10.1002/9781119419310.ch3.

18. MacDonald, Matthew \& Mabbutt, Dan \& Freeman, Adam. (2010). XML. 10.1007/978-1-4302-2512-6_14.

19. Spell, Brett. (2015). Using XML. 10.1007/978-1-48420641-6_14.

20. El-Gazzar, Rania \& Badawy, Osama \& Kholief, M.. (2010). Agent-Based Mobile Event Notification System. International Journal of Interactive Mobile Technologies (iJIM). 4. 10.3991/ijim.v4i4.1427. 